\title{
On the Oscillation of Even-Order Half-Linear Functional Difference Equations with Damping Term
}

\author{
Yaşar Bolat $^{1}$ and Jehad Alzabut ${ }^{2}$ \\ ${ }^{1}$ Department of Mathematics, Faculty of Science and Literatures, Kastamonu University, 037100 Kastamonu, Turkey \\ ${ }^{2}$ Department of Mathematics and Physical Sciences, Prince Sultan University, P.O. Box 66833, Riyadh 11586, Saudi Arabia
}

Correspondence should be addressed to Jehad Alzabut; jalzabut@psu.edu.sa

Received 6 February 2014; Revised 2 May 2014; Accepted 6 May 2014; Published 19 May 2014

Academic Editor: S. R. Grace

Copyright ( 2014 Y. Bolat and J. Alzabut. This is an open access article distributed under the Creative Commons Attribution License, which permits unrestricted use, distribution, and reproduction in any medium, provided the original work is properly cited.

We investigate the oscillatory behavior of solutions of the $m$ th order half-linear functional difference equations with damping term of the form $\Delta\left[p_{n} Q\left(\Delta^{m-1} y_{n}\right)\right]+q_{n} Q\left(\Delta^{m-1} y_{n}\right)+r_{n} Q\left(y_{\tau_{n}}\right)=0, n \geq n_{0}$, where $m$ is even and $Q(s)=|s|^{\alpha-2} s, \alpha>1$ is a fixed real number. Our main results are obtained via employing the generalized Riccati transformation. We provide two examples to illustrate the effectiveness of the proposed results.

\section{Introduction}

Consider the second order half-linear difference equation:

$$
\Delta\left[p_{n}\left|\Delta y_{n}\right|^{\alpha-2} \Delta y_{n}\right]+r_{n}\left|y_{n+1}\right|^{\alpha-2} y_{n+1}=0, \quad n \geq n_{0}, \alpha>1,
$$

where $\Delta$ is the forward difference operator and $\left\{p_{n}\right\},\left\{r_{n}\right\}$ are sequences of nonnegative real numbers with $\left\{p_{n}\right\}>0$. The study of (1) has been initiated by Rehák in [1]. It is well known that there is a close similarity between (1) and the linear second order difference equation. Indeed, if $\left\{y_{n}\right\}$ is a solution of (1), then so is $\left\{c y_{n}\right\}$ for any constant $c$. Thus, (1) has one half of linearity properties [2].

In the presence of damping, (1) has been extended further to the second order half-linear difference equation with damping term of the form

$$
\begin{array}{r}
\Delta\left[p_{n}\left|\Delta y_{n}\right|^{\alpha-2} \Delta y_{n}\right]+q_{n}\left|\Delta y_{n}\right|^{\alpha-2} \Delta y_{n}+r_{n}\left|y_{n+1}\right|^{\alpha-2} y_{n+1}=0, \\
n \geq n_{0} \in \mathbb{N},
\end{array}
$$

where $\left\{q_{n}\right\}$ is a sequence of nonnegative real numbers. It is to be noted that neither (1) nor (2) has involved a delaying term. There are numerous numbers of oscillation criteria established in the literature for the solutions of (1) and (2). Most of these results were obtained by using certain efficient tools among them we name the Riccati transformation, variational principle, and some inequality techniques; see, for instance, the monograph [3] in which many contributions have been cited therein and to the recent papers [4-9].

Let $Q: \mathbb{R} \rightarrow \mathbb{R}$ be defined by $Q(s)=|s|^{\alpha-2} s ; \alpha>1$ is a fixed real number and $\mathbb{N}_{n_{0}}=\left\{n_{0}, n_{0}+1, \ldots\right\}$. Consider the $m$ th order half-linear functional difference equation with damping term of the form

$$
\begin{array}{r}
\Delta\left[p_{n} Q\left(\Delta^{m-1} y_{n}\right)\right]+q_{n} Q\left(\Delta^{m-1} y_{n}\right)+r_{n} Q\left(y_{\tau_{n}}\right)=0, \\
n \in \mathbb{N}_{n_{0}},
\end{array}
$$

where $m$ is even number, and

(H1) $\left\{p_{n}\right\}: \mathbb{N}_{n_{0}} \rightarrow \mathbb{R}^{+}$with $\Delta p_{n} \geq 0$ for all $n \geq n_{0}$;

(H2) $\left\{q_{n}\right\}$ and $\left\{r_{n}\right\}: \mathbb{N}_{n_{0}} \rightarrow \mathbb{R}$ with $q_{n} \geq 0$ and $r_{n}>0$;

(H3) $\left\{\tau_{n}\right\}: \mathbb{N}_{n_{0}} \rightarrow \mathbb{Z}$ with $\tau_{n}<n$ and $\lim _{n \rightarrow \infty} \tau_{n}=\infty$.

For close results regarding the continuous counterparts of (1), (2), and (3), the reader is suggested to consult [10-14].

A primary purpose of this paper is to establish sufficient conditions that guarantee the oscillation of solutions of (3). Our main results are obtained via employing the generalized 
Riccati transformation. In view of (3), one can easily figure out that it is formulated in more general form so that it includes some particular cases which have been studied in the literature; see [15-23] for more details. To the best of authors' observation, however, no published result has been concerned with the investigation of oscillatory behavior of solutions of (3) or its continuous counterpart. Therefore, our paper is new and presents a new approach.

\section{Main Results}

We start by recalling the following standard definitions.

Definition 1. A nontrivial sequence $y_{n}$ is called a solution of (3) if it is defined for all $n \geq \sigma$ where $n \in \mathbb{Z}, \sigma=\min _{i \geq n_{0}}\left\{\tau_{i}\right\}$, and $p_{n} Q\left(\Delta^{m-1} y_{n}\right)$ is differenceable on $\mathbb{N}_{n_{0}}$ and satisfies (3) for all $n \in \mathbb{N}_{n_{0}}$.

Definition 2. A nontrivial solution $y_{n}$ of (3) is said to be oscillatory if the terms of the sequence $y_{n}$ are not eventually positive or not eventually negative. Otherwise, the solution is called nonoscillatory. A difference equation is called oscillatory if all its solutions oscillate.

To obtain our main results, we need the following essential lemmas. The first of these is the discrete analogue of the well-known Kiguradze's lemma.

Lemma 3 (see [24]). Let $y_{n}$ be defined for $n \geq n_{0} \in \mathbb{N}$ and $y(n)>0$ with $\Delta^{m} y_{n}$ of constant sign for $n \geq n_{0}$ and not identically zero. Then, there exists an integer $l, 0 \leq l \leq m$ with $(m+l)$ odd for $\Delta^{m} y_{n} \leq 0$ and $(m+l)$ even for $\Delta^{m} y(n) \geq 0$ such that
(i) $l \leq m-1$ implies $(-1)^{l+i} \Delta^{i} y_{n}>0$ for all $n \geq n_{0}, l \leq i \leq$ $m-1$,

(ii) $l \geq 1$ implies $\Delta^{i} y_{n}>0$ for all large $n \geq n_{0}, 1 \leq i \leq l-1$.

Lemma 4 (see [25]). Let $y_{n}$ be defined for $n \geq n_{0}$ and $y_{n}>0$ with $\Delta^{m} y_{n} \leq 0$ for $n \geq n_{0}$ and not identically zero. Then, there exists a large integer $n_{1} \geq n_{0}$ such that

$$
y_{n} \geq \frac{1}{(m-1) !}\left(n-n_{1}\right)^{m-1} \Delta^{m-1} y_{2^{m-l-1} n}, \quad n \geq n_{1},
$$

where $l$ is defined as in Lemma 3. Further, if $y_{n}$ is increasing, then

$$
y_{n} \geq \frac{1}{(m-1) !}\left(\frac{n}{2^{m-1}}\right)^{m-1} \Delta^{m-1} y_{n}, \quad n \geq 2^{m-1} n_{1} .
$$

Lemma 5. Let $y_{n}$ satisfy conditions of Lemmas 3 and 4 and $\Delta^{m-1} y_{n} \Delta^{m} y_{n} \leq 0$ for $n \geq n_{1} \geq n_{0}$. Further, if $y_{n}$ is increasing, then

$$
\Delta y_{n-k} \geq M n^{m-2} \Delta^{m-1} y_{n}, \quad n \geq n_{1}
$$

where $M=\left(1 /\left((m-1) ! 2^{(m-1)^{2}}\right)\right)>0$.

The proof of Lemma 5 is straightforward and it can be achieved by using the last inequality of Lemma 4 .
Lemma 6. Let $y_{n}$ be an eventually positive solution of (3). If

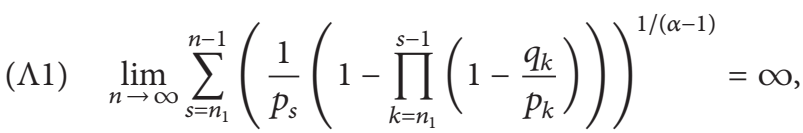

then $\Delta^{m-1} y_{n}>0, \Delta^{m} y_{n} \leq 0$, and $\Delta y_{n}>0$ for all $n \geq n_{1} \geq n_{0}$.

Proof. The fact that $y_{n}$ is eventually positive solution of (3) implies $y_{n}>0$ and $y_{\tau_{n}}>0$ for all $n \geq n_{1} \geq n_{0}$. In view of (3), we get

$$
\Delta\left[p_{n} Q\left(\Delta^{m-1} y_{n}\right)\right]+q_{n} Q\left(\Delta^{m-1} y_{n}\right)<0,
$$

which leads to

$$
\Delta\left[p_{n} Q\left(\Delta^{m-1} y_{n}\right)-\prod_{k=n_{1}}^{n-1}\left(1-\frac{q_{k}}{p_{k}}\right) p_{n_{1}} Q\left(\Delta^{m-1} y_{n_{1}}\right)\right]<0 \text {. }
$$

Hence,

$$
p_{n} Q\left(\Delta^{m-1} y_{n}\right)-\prod_{k=n_{1}}^{n-1}\left(1-\frac{q_{k}}{p_{k}}\right) p_{n_{1}} Q\left(\Delta^{m-1} y_{n_{1}}\right)
$$

is decreasing and $\Delta^{m-1} y_{n}$ is eventually positive or eventually negative.

We claim that

$$
\Delta^{m-1} y_{n}>0, \quad n \geq n_{1}
$$

Assume, on the contrary, that $\Delta^{m-1} y_{n}<0, n \geq n_{1}$. Then, from (10), we obtain

$$
\begin{aligned}
p_{n}\left|\Delta^{m-1} y_{n}\right|^{\alpha-2} \Delta^{m-1} y_{n} & -\prod_{k=n_{1}}^{n-1}\left(1-\frac{q_{k}}{p_{k}}\right) p_{n_{1}}\left|\Delta^{m-1} y_{n_{1}}\right|^{\alpha-2} \Delta^{m-1} y_{n_{1}} \\
\leq & p_{n_{1}}\left|\Delta^{m-1} y_{n_{1}}\right|^{\alpha-2} \Delta^{m-1} y_{n_{1}} \\
& -\prod_{k=n_{1}}^{n_{1}-1}\left(1-\frac{q_{k}}{p_{k}}\right) p_{n_{1}}\left|\Delta^{m-1} y_{n_{1}}\right|^{\alpha-2} \Delta^{m-1} y_{n_{1}},
\end{aligned}
$$

where $\prod_{k=n_{1}}^{n_{1}-1}\left(1-\left(q_{k} / p_{k}\right)\right) p_{n_{1}}\left|\Delta^{m-1} y_{n_{1}}\right|^{\alpha-2} \Delta^{m-1} y_{n_{1}}=0$. Therefore, from (12), we have

$$
\begin{aligned}
p_{n}\left|\Delta^{m-1} y_{n}\right|^{\alpha-2} \Delta^{m-1} y_{n} & \\
& -\prod_{k=n_{1}}^{n-1}\left(1-\frac{q_{k}}{p_{k}}\right) p_{n_{1}}\left|\Delta^{m-1} y_{n_{1}}\right|^{\alpha-2} \Delta^{m-1} y_{n_{1}} \\
\leq & p_{n_{1}}\left|\Delta^{m-1} y_{n_{1}}\right|^{\alpha-2} \Delta^{m-1} y_{n_{1}} \\
& -\prod_{k=n_{1}}^{n_{1}-1}\left(1-\frac{q_{k}}{p_{k}}\right) p_{n_{1}}\left|\Delta^{m-1} y_{n_{1}}\right|^{\alpha-2} \Delta^{m-1} y_{n_{1}} \\
\equiv & -M_{1}^{\alpha-1},
\end{aligned}
$$


where $M_{1}=p_{n_{1}}^{1 /(\alpha-1)}\left|\Delta^{m-1} y_{n_{1}}\right|>0$. It follows that

$$
\left(-\Delta^{m-1} y_{n}\right)^{\alpha-1} \geq \frac{M_{1}^{\alpha-1}}{p_{n}}\left(1-\prod_{k=n_{1}}^{n-1}\left(1-\frac{q_{k}}{p_{k}}\right)\right)
$$

or

$$
\Delta^{m-1} y_{n} \leq-M_{1}\left(\frac{1}{p_{n}}\left(1-\prod_{k=n_{1}}^{n-1}\left(1-\frac{q_{k}}{p_{k}}\right)\right)\right)^{1 /(\alpha-1)} .
$$

Consequently, we obtain

$$
\begin{aligned}
\Delta^{m-2} y_{n} \leq & \Delta^{m-2} y_{n_{1}} \\
& -M_{1} \sum_{s=n_{1}}^{n-1}\left(\frac{1}{p_{s}}\left(1-\prod_{k=n_{1}}^{s-1}\left(1-\frac{q_{k}}{p_{k}}\right)\right)\right)^{1 /(\alpha-1)} .
\end{aligned}
$$

Letting $n \rightarrow \infty$ in the above inequality, one gets $\lim _{n \rightarrow \infty} \Delta^{m-2} y_{n}=-\infty$. Hence, $y_{n}$ is an eventually negative function which contradicts that $y_{n}>0$. Therefore, inequality (11) holds.

From (3), we get

$$
\begin{aligned}
\Delta\left[p_{n} Q\left(\Delta^{m-1} y_{n}\right)\right]= & p_{n+1} \Delta\left(\Delta^{m-1} y_{n}\right)^{\alpha-1} \\
& +\left(\Delta^{m-1} y_{n}\right)^{\alpha-1} \Delta p_{n} \leq 0
\end{aligned}
$$

from which it follows that

$$
\Delta\left(\Delta^{m-1} y_{n}\right)^{\alpha-1} \leq 0, \quad n \geq n_{1} \geq n_{0} .
$$

The above inequality implies that $\left(\Delta^{m-1} y_{n}\right)^{\alpha-1}$ is nonincreasing. Therefore, we can write

$$
\begin{aligned}
& \Delta\left(\Delta^{m-1} y_{n}\right)^{\alpha-1} \\
& =\left(\Delta^{m-1} y_{n+1}\right)^{\alpha-1}-\left(\Delta^{m-1} y_{n}\right)^{\alpha-1} \\
& =\left[\Delta^{m-1} y_{n+1}-\Delta^{m-1} y_{n}\right] \\
& \quad \times\left[\left(\Delta^{m-1} y_{n+1}\right)^{\alpha-2}+\left(\Delta^{m-1} y_{n+1}\right)^{\alpha-3}\left(\Delta^{m-1} y_{n}\right)\right. \\
& \quad+\left(\Delta^{m-1} y_{n+1}\right)^{\alpha-4}\left(\Delta^{m-1} y_{n}\right)^{2} \\
& \left.\quad+\cdots+\left(\Delta^{m-1} y_{n}\right)^{\alpha-2}\right] \leq 0 .
\end{aligned}
$$

Since $\left(\Delta^{m-1} y_{n}\right)^{\alpha-1}$ is nonincreasing and positive, then from the above inequality, we have

$$
\begin{aligned}
& \Delta\left(\Delta^{m-1} y_{n}\right)^{\alpha-1} \\
& \quad \leq\left[\Delta^{m-1} y_{n+1}-\Delta^{m-1} y_{n}\right](\alpha-1)\left(\Delta^{m-1} y_{n}\right)^{\alpha-2} \\
& \quad=(\alpha-1) \Delta\left(\Delta^{m-1} y_{n}\right)\left(\Delta^{m-1} y_{n}\right)^{\alpha-2} \\
& \quad \leq(\alpha-1) \Delta^{m} y_{n}\left(\Delta^{m-1} y_{n}\right)^{\alpha-2} \leq 0
\end{aligned}
$$

by which we have

$$
\Delta^{m} y_{n} \leq 0
$$

In virtue of (21) and Lemma 3, we deduce that since $m$ is even then $l$ is odd. Hence $\Delta y_{n}>0$ for $n \geq n_{1} \geq n_{0}$. The proof is complete.

Theorem 7. Let condition $(\Lambda 1)$ hold. Further, assume that there exists a constant $\lambda>\alpha-1$ such that

$$
\begin{aligned}
& (\Lambda 2) \limsup _{n \rightarrow \infty} \frac{1}{\left(n-n_{1}\right)^{\lambda}} \sum_{k=n_{1}}^{n-1}\left[(n-k)^{\lambda} r_{k}\right. \\
& \left.-\frac{(\alpha-1)^{\alpha-1}}{\alpha^{\alpha}} X_{n k}^{\alpha} Y_{n k}^{1-\alpha}\right] \\
& =\infty,
\end{aligned}
$$

where

$$
\begin{aligned}
& X_{n k}=\left((n+1-k)^{\lambda}-(n-k)^{\lambda}\left(1+\frac{q_{k}}{p_{k+1}}\right)\right), \\
& Y_{n k}=(\alpha-1) M \tau_{k}^{m-2}(n-k)^{\lambda} \frac{p_{k}}{p_{k+1}}>0,
\end{aligned}
$$

and $M$ is as in Lemma 5. Then, (3) is oscillatory.

Proof. For the sake of contradiction, assume that (1) has a nonoscillatory solution $y_{n}$. Without loss of generality, we assume that $y_{n}$ is eventually positive (the proof is similar when $y_{n}$ is eventually negative). That is, $y_{n}>0, y_{\tau_{n}}>0$ and $y_{\tau_{n-k}}>0$ for all $n \geq n_{1} \geq n_{0}$. By Lemma 6, we have $\Delta^{m-1} y_{n}>0, \Delta^{m} y_{n} \leq 0$, and $\Delta y_{n}>0$ for $n \geq n_{1}$. Consider the function

$$
w_{n}=\frac{p_{n} Q\left(\Delta^{m-1} y_{n}\right)}{Q\left(y_{\tau_{n-k}}\right)}=p_{n}\left(\frac{\Delta^{m-1} y_{n}}{y_{\tau_{n-k}}}\right)^{\alpha-1}>0, \quad n \geq n_{1} .
$$

Taking into account that $\Delta y_{n}>0$ and $y_{n}$ is increasing and $\tau_{n-k}<\tau_{n}$, we deduce that $\Delta^{m} y_{n} \leq 0$ and $\Delta^{m-1} y_{n}$ is nonincreasing. Lemmas 3 and 4, (1), and (24) yield

$$
\begin{aligned}
\Delta w_{n}= & \frac{-q_{n}\left(\Delta^{m-1} y_{n}\right)^{\alpha-1}-r_{n}\left(y_{\tau_{n}}\right)^{\alpha-1}}{\left(y_{\tau_{n+1-k}}\right)^{\alpha-1}} \\
& -\frac{p_{n}\left(\Delta^{m-1} y_{n}\right)^{\alpha-1} \Delta\left(y_{\tau_{n-k}}\right)^{\alpha-1}}{\left(y_{\tau_{n-k}}\right)^{\alpha-1}\left(y_{\tau_{n+1-k}}\right)^{\alpha-1}} \\
\leq & -r_{n}-\frac{q_{n}}{p_{n+1}} w_{n+1} \\
& -\frac{(\alpha-1) p_{n}\left(\Delta^{m-1} y_{n}\right)^{\alpha-1}\left(y_{\tau_{n-k}}\right)^{\alpha-2} \Delta y_{\tau_{n-k}}}{\left(y_{\tau_{n-k}}\right)^{\alpha-1}\left(y_{\tau_{n+1-k}}\right)^{\alpha-1}}
\end{aligned}
$$




$$
\begin{aligned}
\leq & -r_{n}-\frac{q_{n}}{p_{n+1}} w_{n+1} \\
& -\frac{(\alpha-1) p_{n}\left(\Delta^{m-1} y_{n}\right)^{\alpha-1}\left(y_{\tau_{n-k}}\right)^{\alpha-2} M \tau_{n}^{m-2} \Delta^{m-1} y_{\tau_{n}}}{\left(y_{\tau_{n-k}}\right)^{\alpha-1}\left(y_{\tau_{n+1-k}}\right)^{\alpha-1}} \\
\leq & -r_{n}-\frac{q_{n}}{p_{n+1}} w_{n+1}-\frac{(\alpha-1) p_{n}\left(\Delta^{m-1} y_{n}\right)^{\alpha} M \tau_{n}^{m-2}}{\left(y_{\tau_{n+1-k}}\right)^{\alpha}} \\
\leq & -r_{n}-\frac{q_{n}}{p_{n+1}} w_{n+1}-\frac{(\alpha-1) M \tau_{n}^{m-2} p_{n}\left(\Delta^{m-1} y_{n+1}\right)^{\alpha}}{\left(y_{\tau_{n+1-k}}\right)^{\alpha}} \\
= & -r_{n}-\frac{q_{n}}{p_{n+1}} w_{n+1} \\
& -\frac{p_{n}(\alpha-1) M \tau_{n}^{m-2} p_{n+1}\left(\Delta^{m-1} y_{n+1}\right)^{\alpha}}{p_{n+1}\left(y_{\tau_{n+1-k}}\right)^{\alpha}} \\
= & -r_{n}-\frac{q_{n}}{p_{n+1}} w_{n+1}-(\alpha-1) M \tau_{n}^{m-2} \frac{p_{n}}{p_{n+1}} w_{n+1}^{\alpha /(\alpha-1)} .
\end{aligned}
$$

Multiplying by $(n-k)^{\lambda}$ and summing up from $n_{1}$ to $n-1$, we obtain

$$
\begin{aligned}
& \sum_{k=n_{1}}^{n-1}(n-k)^{\lambda} r_{k} \leq-\sum_{k=n_{1}}^{n-1}(n-k)^{\lambda} \Delta w_{k} \\
& -\sum_{k=n_{1}}^{n-1}(n-k)^{\lambda} \frac{q_{k}}{p_{k+1}} w_{k+1} \\
& -\sum_{k=n_{1}}^{n-1}(\alpha-1) M \tau_{k}^{m-2}(n-k)^{\lambda} \frac{p_{k}}{p_{k+1}} w_{k+1}^{\alpha /(\alpha-1)} \\
& \leq\left(n-n_{1}\right)^{\lambda} w_{n_{1}}-w_{n} \\
& +\sum_{k=n_{1}}^{n-1}\left((n+1-k)^{\lambda}\right. \\
& \left.-(n-k)^{\lambda}\left(1+\frac{q_{k}}{p_{k+1}}\right)\right) w_{k+1} \\
& -\sum_{k=n_{1}}^{n-1}(\alpha-1) M \tau_{k}^{m-2}(n-k)^{\lambda} \frac{p_{k}}{p_{k+1}} w_{k+1}^{\alpha /(\alpha-1)} \\
& \leq\left(n-n_{1}\right)^{\lambda} w_{n_{1}} \\
& +\sum_{k=n_{1}}^{n-1}\left((n+1-k)^{\lambda}\right. \\
& \left.-(n-k)^{\lambda}\left(1+\frac{q_{k}}{p_{k+1}}\right)\right) w_{k+1} \\
& -\sum_{k=n_{1}}^{n-1}(\alpha-1) M \tau_{k}^{m-2}(n-k)^{\lambda} \frac{p_{k}}{p_{k+1}} w_{k+1}^{\alpha /(\alpha-1)}
\end{aligned}
$$

or

$$
\begin{aligned}
& \frac{1}{\left(n-n_{1}\right)^{\lambda}}\left[\sum_{k=n_{1}}^{n-1}(n-k)^{\lambda} r_{k}-\sum_{k=n_{1}}^{n-1}\left(X_{n k} w_{k+1}-Y_{n k} w_{k+1}^{\alpha /(\alpha-1)}\right)\right] \\
& \quad \leq w_{n_{1}},
\end{aligned}
$$

where

$$
\begin{aligned}
& X_{n k}=\left((n+1-k)^{\lambda}-(n-k)^{\lambda}\left(1+\frac{q_{k}}{p_{k+1}}\right)\right), \\
& Y_{n k}=(\alpha-1) M \tau_{k}^{m-2}(n-k)^{\lambda} \frac{p_{k}}{p_{k+1}}>0 .
\end{aligned}
$$

Let

$$
F\left(w_{k+1}\right)=X_{n k} w_{k+1}-Y_{n k} w_{k+1}^{\alpha /(\alpha-1)}
$$

Then, $F$ has maximum value at $w_{k+1}=((\alpha-1) / \alpha)^{\alpha-1} X_{n k}^{\alpha-1} Y_{n k}^{1-\alpha}$. That is,

$$
F_{\max }=\frac{(\alpha-1)^{\alpha-1}}{\alpha^{\alpha}} X_{n k}^{\alpha} Y_{n k}^{1-\alpha}
$$

Therefore, (27) can be rewritten as

$$
\frac{1}{\left(n-n_{1}\right)^{\lambda}} \sum_{k=n_{1}}^{n-1}\left[(n-k)^{\lambda} r_{k}-\frac{(\alpha-1)^{\alpha-1}}{\alpha^{\alpha}} X_{n k}^{\alpha} Y_{n k}^{1-\alpha}\right] \leq w_{n_{1}}
$$

Hence, we have

$$
\begin{aligned}
& \limsup _{n \rightarrow \infty} \frac{1}{\left(n-n_{1}\right)^{\lambda}} \sum_{k=n_{1}}^{n-1}\left[(n-k)^{\lambda} r_{k}-\frac{(\alpha-1)^{\alpha-1}}{\alpha^{\alpha}} X_{n k}^{\alpha} Y_{n k}^{1-\alpha}\right] \\
& \quad \leq w_{n_{1}}
\end{aligned}
$$

which contradicts condition $(\Lambda 2)$. The proof is complete.

Theorem 8. Let condition $(\Lambda 1)$ hold. Further, assume that there exists a function $\delta_{n}: \mathbb{N} \rightarrow \mathbb{R}^{+}$such that

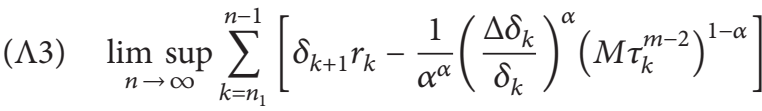

$$
\begin{aligned}
& =\infty, \quad n_{1} \geq n_{0} \text {, }
\end{aligned}
$$

where $M$ is as in Lemma 5. Then, (3) is oscillatory.

Proof. For the sake of contradiction, assume that (3) has a nonoscillatory solution $y_{n}$. Without loss of generality, we assume that $y_{n}$ is eventually positive (the proof is similar when $y_{n}$ is eventually negative). That is, $y_{n}>0, y_{\tau_{n}}>0$ and $y_{\tau_{n-k}}>0$ for all $n \geq n_{1} \geq n_{0}$. By Lemma 6, we have 
$\Delta^{m-1} y_{n}>0, \Delta^{m} y_{n} \leq 0$, and $\Delta y_{n}>0$ for $n \geq n_{1}$. Consider the function

$$
w_{n}=\delta_{n} p_{n}\left(\frac{\Delta^{m-1} y_{n}}{y_{\tau_{n-k}}}\right)^{\alpha-1}>0, \quad n \geq n_{1} .
$$

By utilizing the same approach as in the proof of Theorem 7, we arrive at

$$
\Delta w_{n} \leq-\delta_{n+1} r_{n}+\frac{1}{\alpha^{\alpha}}\left(\frac{\Delta \delta_{n}}{\delta_{n}}\right)^{\alpha}\left(M \tau_{n}^{m-2}\right)^{1-\alpha} .
$$

Summing up (35) from $n_{1}$ to $n-1$, we have

$$
\sum_{k=n_{1}}^{n-1}\left[\delta_{k+1} r_{k}-\frac{1}{\alpha^{\alpha}}\left(\frac{\Delta \delta_{k}}{\delta_{k}}\right)^{\alpha}\left(M \tau_{k}^{m-2}\right)^{1-\alpha}\right] \leq w_{n_{1}} .
$$

Letting $n \rightarrow \infty$ in the above inequality and taking the upper limit, we get a contradiction to $(\Lambda 3)$. The proof is complete.

Remark 9. In view of the statements of Theorems 7 and 8 , one can easily deduce that condition $(\Lambda 3)$ is a generalization of $(\Lambda 2)$.

Example 10. Consider the fourth order half-linear functional difference equation with damping

$$
\Delta\left[n\left(\Delta^{3} y_{n}\right)^{2}\right]+n\left(\Delta^{3} y_{n}\right)^{2}+\frac{1}{n} y_{n-1}^{2}=0, \quad n \geq 2
$$

where $p_{n}=n, q_{n}=n, r_{n}=1 / n, \tau_{n}=n-1, m=4$, and $\alpha=3$. It is easy to see that conditions (H1)-(H3) are satisfied. It remains to check the validity of conditions $\Lambda 1$ and $\Lambda 2$.

For $n \geq 2$, we have

$$
\Gamma 1:=\sum_{s=n_{1}}^{n-1}\left(\frac{1}{p_{s}}\left(1-\prod_{\nu=n_{1}}^{s-1}\left(1-\frac{q_{\nu}}{p_{\nu}}\right)\right)\right)^{1 /(\alpha-1)}=\sum_{s=2}^{n-1}\left(\frac{1}{s}\right)^{1 / 2} .
$$

It is clear that $\Gamma 1 \rightarrow \infty$ as $n \rightarrow \infty$. Therfore, condition $(\Lambda 1)$ holds. For $n \geq 2$ and $\lambda=3>\alpha-1=2$, we have

$$
\begin{aligned}
\Gamma 2 & :=\frac{1}{\left(n-n_{1}\right)^{\lambda}} \sum_{k=n_{1}}^{n-1}\left[(n-k)^{\lambda} r_{k}-\frac{(\alpha-1)^{\alpha-1}}{\alpha^{\alpha}} X_{n k}^{\alpha} Y_{n k}^{1-\alpha}\right] \\
& =\frac{1}{(n-2)^{3}} \sum_{k=2}^{n-1}\left[\frac{(n-k)^{3}}{k}-\frac{4}{27} X_{n k}^{3} Y_{n k}^{2}\right]
\end{aligned}
$$

where

$$
\begin{aligned}
X_{n k}^{3} & =\left[\frac{(k+1)(n+1-k)^{3}-(2 k+1)(n-k)^{3}}{k+1}\right]^{3}, \\
Y_{n k}^{2} & =\frac{(k+1)^{2}}{4 M^{2} k^{2}(k-2)^{8}(n-k)^{6}} .
\end{aligned}
$$

It is clear that $\Gamma 2 \rightarrow \infty$ as $n \rightarrow \infty$. Then, condition $(\Lambda 2)$ holds. Thus, by the conclusion of Theorem $7,(37)$ is oscillatory.
Example 11. Consider the sixth order half-linear functional difference equation with damping

$$
\Delta\left[n\left(\Delta^{5} y_{n}\right)^{2}\right]+n\left(\Delta^{5} y_{n}\right)^{2}+n^{2} y_{n-1}^{2}=0, \quad n \geq 2,
$$

where $p_{n}=n, q_{n}=n, r_{n}=n^{2}, \tau_{n}=n-1, m=6$, and $\alpha=3$. It is easy to see that conditions (H1)-(H3) are satisfied. In Example 10, we have seen that $(\Lambda 1)$ is satisfied. It remains to check the validity of condition $(\Lambda 3)$.

For $n \geq 2$ and $\delta_{n}=n$, we have

$$
\begin{aligned}
\Gamma 3 & :=\sum_{k=n_{1}}^{n-1}\left[\delta_{k+1} r_{k}-\frac{1}{\alpha^{\alpha}}\left(\frac{\Delta \delta_{k}}{\delta_{k}}\right)^{\alpha}\left(M \tau_{k}^{m-2}\right)^{1-\alpha}\right] \\
& =\sum_{k=2}^{n-1}\left[k^{2}(k+1)-\frac{1}{27 M^{2} k^{3}(k-1)^{8}}\right] \\
& =\sum_{k=2}^{n-1}\left[\frac{27 M^{2} k^{5}(k+1)(k-1)^{8}-1}{27 M^{2} k^{3}(k-1)^{8}}\right] .
\end{aligned}
$$

It is clear that $\mathrm{\Gamma} 3 \rightarrow \infty$ as $n \rightarrow \infty$. Then, condition $(\Lambda 3)$ holds. Thus, by the conclusion of Theorem $8,(41)$ is oscillatory.

Remark 12. It is not possible to decide the oscillatory behavior of solutions of (37) and (41) by using any of the results reported in $[12,13]$. This implies that the results of our paper extend and generalize some known theorems.

Remark 13. The main results of this paper remain valid for nondelay difference equations of the form

$$
\begin{array}{r}
\Delta\left[p_{n} Q\left(\Delta^{m-1} y_{n}\right)\right]+q_{n} Q\left(\Delta^{m-1} y_{n}\right)+r_{n} Q\left(y_{n}\right)=0, \\
n \in \mathbb{N}_{n_{0}} .
\end{array}
$$

\section{Conflict of Interests}

The authors declare that there is no conflict of interests regarding the publication of this paper.

\section{Acknowledgment}

The authors would like to express thier sincere thanks to the referee for pointing out several suggestions and corrections that helped making the contents of this paper more accurate.

\section{References}

[1] P. Rehák, "Oscillatory properties of second order half-linear difference equations," Czechoslovak Mathematical Journal, vol. 51, no. 126, pp. 303-321, 2001.

[2] O. Došlý and P. Rehák, Half-Linear Differential Equations, vol. 202 of Mathematics Studies, North-Holland, Amsterdam, The Netherlands, 2005.

[3] R. Agarwal, M. Bohner, S. R. Grace, and D. O'Regan, Discrete Oscillation Theory, Hindawi Publishing Corporation, New York, NY, USA, 2005. 
[4] M. Cecchi, Z. Došlá, M. Marini, and I. Vrkoč, "Asymptotic properties for half-linear difference equations," Mathematica Bohemica, vol. 131, no. 4, pp. 347-363, 2006.

[5] Y. G. Sun and F. W. Meng, "Nonoscillation and oscillation of second order half-linear difference equations," Applied Mathematics and Computation, vol. 197, no. 1, pp. 121-127, 2008.

[6] J. Jiang and X. Tang, "Oscillation of second order half-linear difference equations (II)," Applied Mathematics Letters, vol. 24, no. 9, pp. 1495-1501, 2011.

[7] O. Došlý and S. Fišnarová, "Linearized Riccati technique and (non-)oscillation criteria for half-linear difference equations," Advances in Difference Equations, vol. 2008, Article ID 438130, 18 pages, 2008.

[8] O. Došlý and S. Fišnarová, "Perturbation principle in discrete half-linear oscillation theory," Studies of the University of Žilina. Mathematical Series, vol. 23, no. 1, pp. 19-28, 2009.

[9] O. Došlý and S. Fišnarová, "Variational technique and principal solution in half-linear oscillation criteria," Applied Mathematics and Computation, vol. 217, no. 12, pp. 5385-5391, 2011.

[10] R. P. Agarwal, S. R. Grace, and D. O'Regan, Oscillation Theory for Second Order Linear, Half-Linear, Superlinear and Sublinear Dynamic equations, Kluwer Academic Publishers, Dordrecht, The Netherlands, 2002.

[11] O. Došlý and A. Lomtatidze, "Oscillation and nonoscillation criteria for half-linear second order differential equations," Hiroshima Mathematical Journal, vol. 36, no. 2, pp. 203-219, 2006.

[12] S. Liu, Q. Zhang, and Y. Yu, "Oscillation of even-order halflinear functional differential equations with damping," Computers \& Mathematics with Applications, vol. 61, no. 8, pp. 21912196, 2011.

[13] Q. Zhang, S. Liu, and L. Gao, "Oscillation criteria for even-order half-linear functional differential equations with damping," Applied Mathematics Letters, vol. 24, no. 10, pp. 1709-1715, 2011.

[14] C. Zhang, T. Li, B. Sun, and E. Thandapani, "On the oscillation of higher-order half-linear delay differential equations," Applied Mathematics Letters, vol. 24, no. 9, pp. 1618-1621, 2011.

[15] M. Cecchi, Z. Došlá, and M. Marini, "Positive decreasing solutions of quasi-linear difference equations," Computers \& Mathematics with Applications, vol. 42, no. 10-11, pp. 1401-1410, 2001.

[16] O. Došlý and P. Rehák, "Nonoscillation criteria for half-linear second-order difference equations," Computers \& Mathematics with Applications, vol. 42, no. 3-5, pp. 453-464, 2001, Advances in difference equations, III.

[17] P. Rehák, "Generalized discrete Riccati equation and oscillation of half-linear difference equations," Mathematical and Computer Modelling, vol. 34, no. 3-4, pp. 257-269, 2001.

[18] P. Rehák, "Oscillation and nonoscillation criteria for second order linear difference equations," Fasciculi Mathematici, vol. 31, pp. 71-89, 2001.

[19] E. Thandapani, M. M. S. Manuel, J. R. Graef, and P. W. Spikes, "Monotone properties of certain classes of solutions of secondorder difference equations," Computers \& Mathematics with Applications, vol. 36, no. 10-12, pp. 291-297, 1998, Advances in difference equations, II.

[20] E. Thandapani and K. Ravi, "Bounded and monotone properties of solutions of second-order quasilinear forced difference equations," Computers \& Mathematics with Applications, vol. 38, no. 2, pp. 113-121, 1999.
[21] E. Thandapani and K. Ravi, "Oscillation of second-order halflinear difference equations," Applied Mathematics Letters, vol. 13, no. 2, pp. 43-49, 2000.

[22] S. H. Saker, "Oscillation criteria of second-order half-linear delay difference equations," Kyungpook Mathematical Journal, vol. 45, no. 4, pp. 579-594, 2005.

[23] Y. Bolat and J. O. Alzabut, "On the oscillation of higher-order half-linear delay difference equations," Applied Mathematics \& Information Sciences, vol. 6, no. 3, pp. 423-427, 2012.

[24] R. P. Agarwal and S. R. Grace, "Oscillation of certain functionaldifferential equations," Computers \& Mathematics with Applications, vol. 38, no. 5-6, pp. 143-153, 1999.

[25] M. Migda, "On the discrete version of generalized Kiguradze's lemma," Fasciculi Mathematici, vol. 35, pp. 1-7, 2005. 


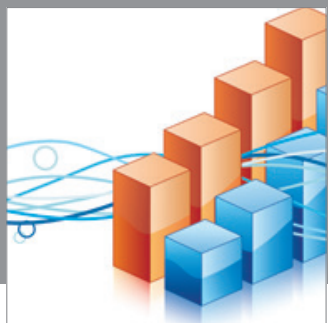

Advances in

Operations Research

mansans

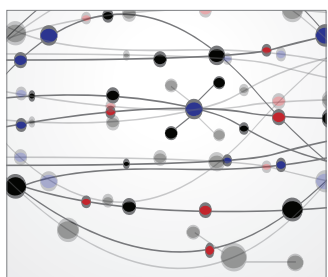

The Scientific World Journal
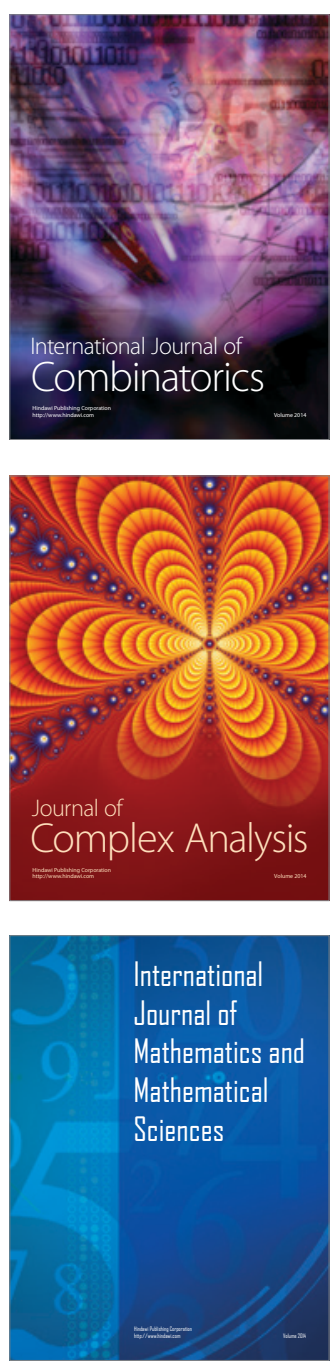
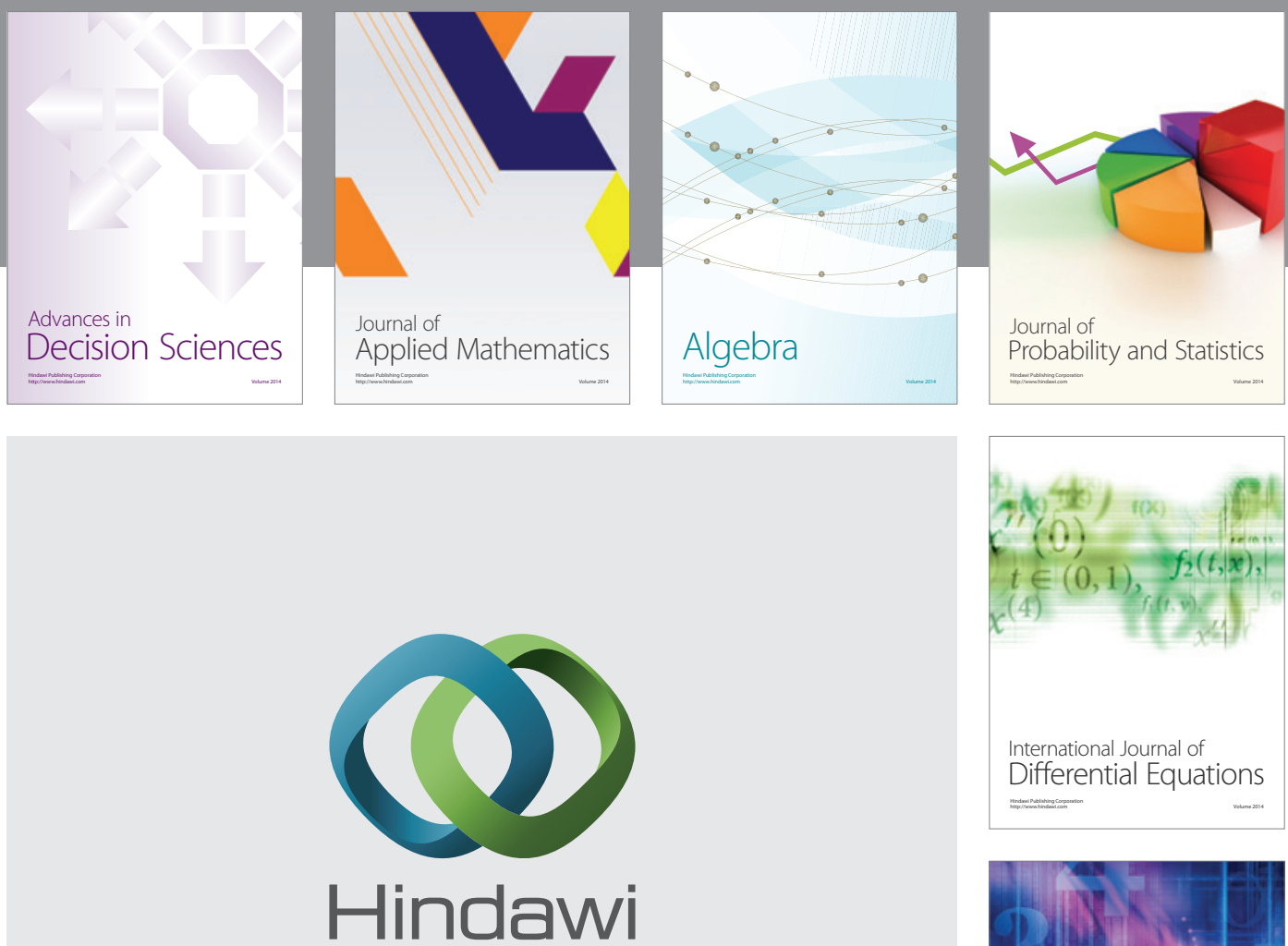

Submit your manuscripts at http://www.hindawi.com
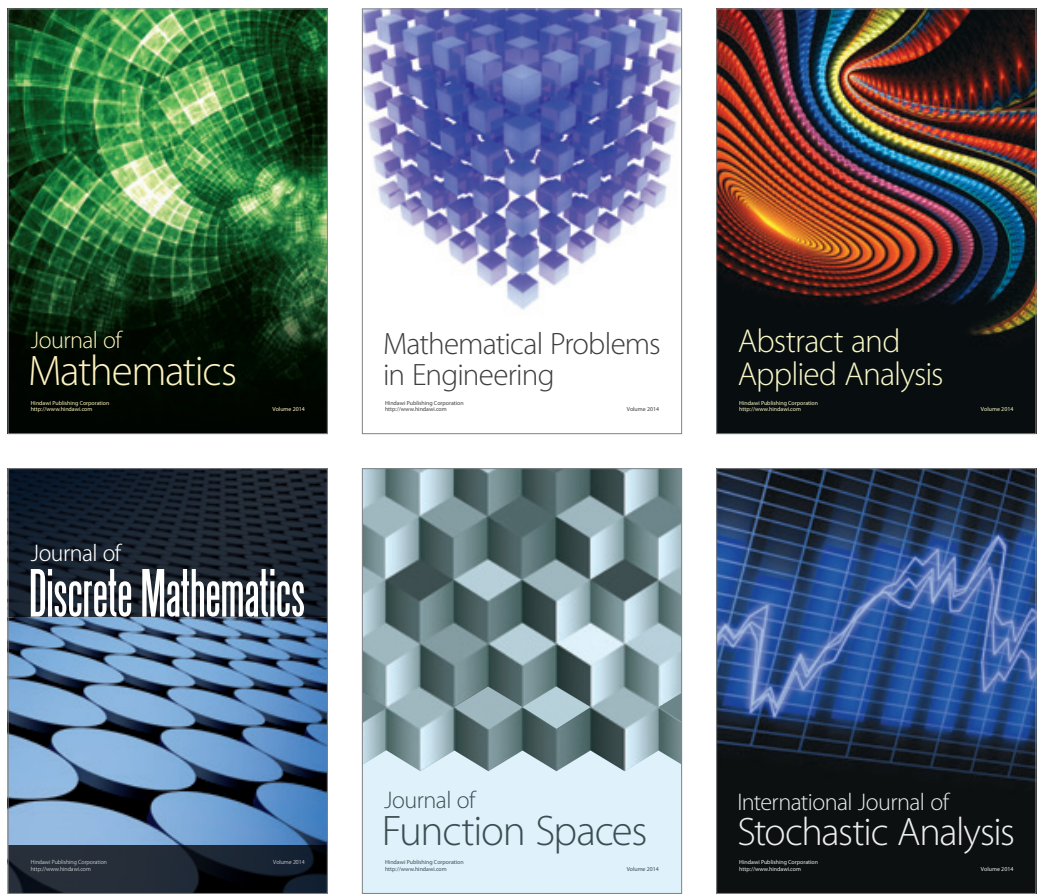

Journal of

Function Spaces

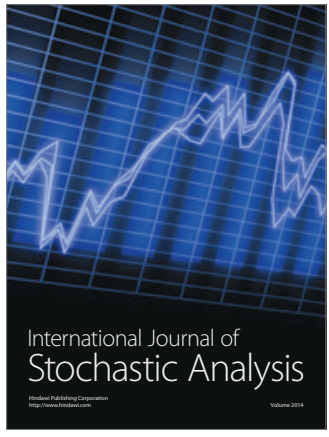

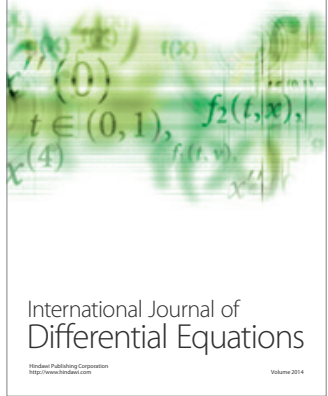
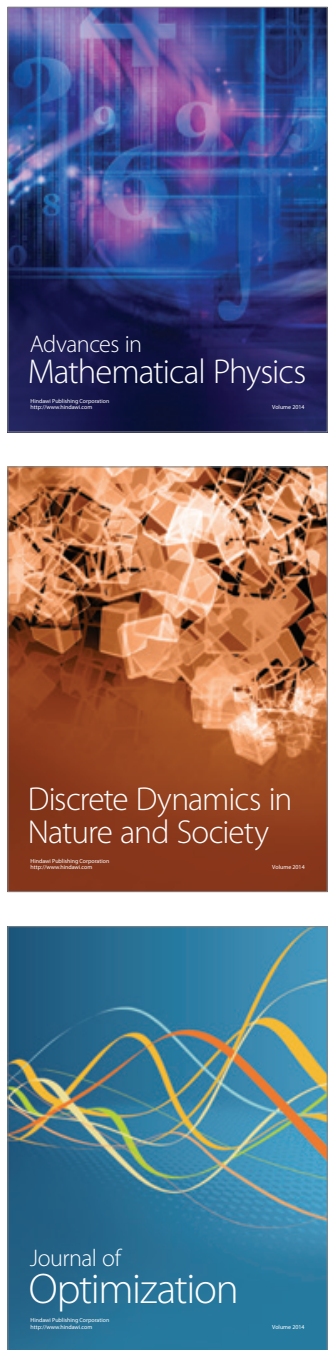